\title{
GMR
}

\section{Decreased expression of humanized Fat-1 in porcine fetal fibroblasts following deletion of PGK-neomycin resistance}

\author{
X.J. Han ${ }^{1 *}$, H. Liang ${ }^{1 *}$, T. Yun ${ }^{1}$, Y.H. Zhao ${ }^{1}$, M.L. Zhang ${ }^{2,3}$, L.H. Zhao ${ }^{2,3}$, \\ R.F. $\mathrm{Li}^{2,3}$ and X.L. $\mathbf{L i}^{1}$ \\ ${ }^{1}$ Key Laboratory of National Education Ministry for Mammalian \\ Reproductive Biology and Biotechnology, Inner Mongolia University, \\ Yu Quan Qu, Hohhot, Inner Mongolia, China \\ ${ }^{2}$ State Key Laboratories of Reproductive Medicine, Nanjing Medical University, \\ Nanjing, China \\ ${ }^{3}$ Jiangsu Key Laboratory of Xenotransplantation, Nanjing Medical University, \\ Nanjing, China \\ *These authors contributed equally to this study. \\ Corresponding author: R.F. Li / X.L. Li \\ E-mail: lirongfeng@njmu.edu.cn / lixueling@hotmail.com
}

Genet. Mol. Res. 14 (3): 11594-11604 (2015)

Received February 3, 2015

Accepted May 14, 2015

Published September 28, 2015

DOI http://dx.doi.org/10.4238/2015.September.28.11

\begin{abstract}
The neomycin-resistance $\left(\right.$ neo $\left.^{\mathrm{r}}\right)$ gene is widely used as a selectable marker in eukaryotic expression vectors; however, its expression often affects that of target genes. Cre recombinase recognizes LoxP sites, leading to site-specific recombination and deletion of DNA and RNA between two LoxP sites. In the present study, a humanized Fat-1 gene (hFat-1) was generated by DNA Works and used to construct a pC-PGK-neo ${ }^{\mathrm{r}}$-hfat-1 expression vector, in which PGK-neor was flanked by two loxP sites. The pC-PGKneo ${ }^{\mathrm{r}}$-hfat-1 plasmids were transfected into porcine fetal fibroblasts using liposomes, and three transgenic cell lines were obtained by culturing with $400 \mu \mathrm{g} / \mathrm{mL}$ G418 for 7 days. Next, these cell lines
\end{abstract}


were transfected with a Cre recombinase expression plasmid, which contains a puromycin resistance gene, in order to delete neo ${ }^{\mathrm{r}}$, which was integrated into the genome. hFat-1-neo ${ }^{\mathrm{r}}$ negative cells were obtained following puromycin selection. Real-time quantitative polymerase chain reaction data indicated that neomycin-resistant cells had higher hFat-1 expression than neomycin-sensitive cells. High performance gas chromatography data suggested that the $n-6 / n-3$ ratio was significantly lower in transfected cells than in wild-type cells. The n-6/n-3 ratio in Cre-treated hFat-1-transfected cells was higher than that in untreated cells, suggesting that deletion of PGK-neo ${ }^{r}$ decreased hFat-1 expression.

Key words: Porcine fetal fibroblasts; Cre recombinase; Transfection; hFat-1; PGK-neomycin resistance

\section{INTRODUCTION}

Neomycin-resistance $\left(\right.$ neo $\left.^{\mathrm{r}}\right)$ is widely used as a marker of positive selection in eukaryotic expression vectors; however, its expression often affects that of target genes. PGK-neo ${ }^{r}$ contains a neomycin phosphotransferase gene driven by the phosphoglycerate kinase I promoter, and is used in embryonic stem cells as a selection marker during homologous recombination. When embryonic stem cells containing PGK-neo ${ }^{\mathrm{r}}$ were injected into blastocysts, the PGK-neo ${ }^{r}$ was retained, leading to the generation of fertilized chimeric animals (Scacheri et al., 2001). However, an animal model containing PGK-neo ${ }^{\mathrm{r}}$ would present unexpected phenotypes, since previous studies have shown that cassettes used for positive selection can interfere with the expression of neighboring genes (Hug et al., 1996; Pham et al., 1996). In the context of genetic-tagging studies, such interference could lead to misexpression of the reporter gene, resulting in misidentification of cell types that are identified by the reporter. Intragenic insertion of PGK-neo ${ }^{\mathrm{r}}$ can alter target gene transcription and expression (Holzenberger et al., 2000). PGK-neo ${ }^{\mathrm{r}}$ exerts a positional effect on the adjacent gene, especially when it is inserted into a gene cluster or gene regulation motif. Scacheri et al. (2001) deleted the multiple endocrine neoplasia 1 (MEN1) gene in mouse stem cells using homologous recombination. A total of 2-4 MEN1 exons were replaced with PGK-neo ${ }^{\mathrm{r}}$, reversed, and inserted into the reading frame of MEN1 (Men IMSK/+). Heterologous deletion of MENI resulted in the embryonic lethality of omphalocele at the late stages of pregnancy. Northern blotting analysis revealed a large quantity of abnormal transcription products, and real-time quantitative polymerase chain reaction (RT-PCR) suggested that abnormal transcription was induced by the antisense strand of the PGK promoter.

In general, deletion of the positive selection cassette requires these sequences to be flanked by either LoxP or FRT sequences, which enables their deletion by transient expression of the site-specific recombinases, Cre or Flp, respectively. Deletion of the selection marker can be confirmed by PCR analysis or sensitivity of the ESCs to the selecting agent (Branda and Dymecki, 2004; Schnutgen et al., 2006). This is a routine procedure used in transgenic studies in which mouse and human embryonic stem cells are manipulated (Costa et al., 2005; Davis et al., 2008). However, the effect of PGK-neo ${ }^{r}$ insertion and deletion in transgenic studies in 
domestic animals is rarely reported.

The Fat-1 gene, which was obtained from Caenorhabditis elegans, encodes n-3 fatty acid desaturase, which regulates the production of n-3 polyunsaturated fatty acids (PUFAs) from 18-20-carbon (C) n-6 PUFAs through a dehydrogenation reaction (Spychalla et al., 1997). In this study, the Fat-1 gene was humanized (hFat-1) by DNA Works and used to construct a pCAGG-hfat-1-PGK-neo ${ }^{r}$ expression vectors, which were then transfected into porcine fetal fibroblasts (PFFs) using liposomes to generate transgenic cells. Next, the resistance gene (neo) was deleted from hFat-1 transgenic cells. High performance gas chromatography data showed that the n-6/n-3 ratio was significantly lower in transfected cells than in wild-type cells, and the $n-6 / n-3$ ratio in Cre-treated hFat-1-transfected cells was higher than that in untreated cells, suggesting that deletion of PGK-neo ${ }^{r}$ decreased the expression of hFat-1.

\section{MATERIAL AND METHODS}

\section{Materials}

Mini plasmid extraction kits and agarose gel DNA extraction kits were purchased from Tiangen Co., Ltd. (Beijing, China). PCR primers were synthesized by Invitrogen Co. (Carlsbad, CA, USA). The endonucleases MluI, DraIII, BstBI, NdeI, and NheI were obtained from New England Biosciences (Ipswich, MA, USA), and BamHI and $X b a$ I were obtained from TaKaRa (Dalian, China). Dulbecco's minimum Eagle medium (DMEM) containing high glucose, $0.25 \%$ trypsin, penicillin, and streptomycin was purchased from Gibco (Grand Island, NY, USA). Standard fetal bovine serum and Dulbecco phosphate-buffered saline were obtained from Hyclone (Logan, UT, USA). Additionally, the following were purchased from Corning (Corning, NY, USA): 6-, 12-, and 24-well plates; 60- and 100-mm culture dishes; and 2-mL tubes.

\section{pCAGDNA3 modification and pC-PGK-neo ${ }^{\mathrm{r}}$-pA construction}

The upstream sequence of CAGG (253-bp) was amplified. MluI, PacI, FseI, and SfiI sites were added to the sense primers, and an NdeI site was added to the antisense primers. The sense and antisense primers were as follows: 5'-GACGCGTTTAATTAAGGCCGGCCTT TGGCCATTGAGGCCGTTGACATTGATTATTGACTAG-3' (sequentially, the underlined regions indicate $M l u \mathrm{I}, P a c \mathrm{I}, F s e \mathrm{I}$, and SfiI sites), and 5'-CCATATGATACACTTGATGTA CT-3' (underlined region indicates an NdeI site). The pCAGDNA3 plasmids and PCR products, MluI-PacI-FseI-Sfi-CAGG (upstream $253 \mathrm{bp}$ )-NdeI were double digested with MluI and NdeI. The digested PCR products were ligated to a pCAGDNA3 backbone, and the recombinant plasmids were sequenced. LoxP-PGK-neo ${ }^{\mathrm{r}}-\mathrm{BGHpA}$-LoxP was also amplified by PCR, the sense and antisense primers were as follows: 5'-CACGTAGTGAAGCTTATAACT TCGTATAGCATACATTATACGAAGTTAT-3' (sequentially, the underlined regions indicate DraIII and HindIII sites), and AAGTTAACGCTAGCATAACTTCGTATAATGTATGCTAT ACGAAGTTATTCCCCAGCATGCCTGCTATT-3' (sequentially, the underlined regions indicate NheI, HpaI, and BstBI sites). The length of PCR products was $1.69 \mathrm{~kb}$. Next, the pCAGDNA3-CAGG plasmids and PCR products were double digested with DraIII and BstBI, and the PCR products were ligated to pCAGDNA3-CAGG and named pC-PGK-neor-pA. The recombinant plasmids were sequenced and identified with endonucleases. 


\section{Synthesis of a humanized hFat-1 sequence and construction of pC-PGK-neo ${ }^{\mathrm{r}}$-hfat-1}

The Fat-1 gene of C. elegans was humanized by DNAWorks (http://helixweb.nih.gov/ dnaworks/). The humanized hFat-1 sequence was synthesized and inserted into PUC57CS (Generay, Shanghai, China), and the BamHI site was added to both ends of this gene. pC-PGK-neorpA and PUC57CS-hFat-1 were digested with $B a m H I$, and the digested fragments were ligated and named $\mathrm{pC}-\mathrm{PGK}-$ neo $^{\mathrm{r}}$-hfat-1. The positive clones were identified using endonucleases.

\section{Transfection of porcine fetal fibroblasts using liposomes}

The cells were transfected using Lipofectamine ${ }^{\circledR}$ LTX and PlusT ${ }^{\mathrm{M}}$ Reagent (Cat. No. 15338-100, Invitrogen). The cells were cultured on 24-well plates, and 600 ng linearized expression vector was mixed with $3 \mu \mathrm{L}$ liposomes. Serum-free medium was used to increase the well volume to $100 \mu \mathrm{L}$, and the mixture was incubated at room temperature for $30 \mathrm{~min}$. The media was removed from each well, and the wells were washed with PBS twice. Next, 200 $\mu \mathrm{L}$ serum-free medium was added into each well, along with $100 \mu \mathrm{L}$ prepared mixture. The medium was replaced after $6 \mathrm{~h}$ of culture, and the cells were cultured at $37^{\circ} \mathrm{C}$ in a humidified atmosphere of $5 \% \mathrm{CO}_{2}$. Stably transfected cells were screened by culturing with G418 for 7 days after $48 \mathrm{~h}$ of culture.

\section{Identification of transgenic porcine fetal fibroblasts by PCR and reverse transcription PCR}

To identify the hFat1 gene in transgenic PFFs, we designed four pairs of PCR primers to amplify two parts of the hFat 1 gene. The primers used to amplify the forward fragment were as follows: sense, 5'-CACTACCGAGGAGCCCAGGATACAG-3'; antisense, 5'-TGCGAAAAAG TCG TAGTTCACCTGG-3'. The size of the PCR product was $976 \mathrm{bp}$. The primers used to amplify the reverse fragment were as follows: sense, 5'-ATCCGCTATCTTGTTCAGGACTTCG-3'; antisense, 5'-AGAAGCCATAGAGCCCACCGCATCC-3'. The size of the PCR product was $1349 \mathrm{bp}$. The PCR products were ligated to pMD19-T simple, and sequenced.

For reverse transcription-PCR, total RNA was extracted from hFat-1 transgenic PFFs and reverse transcribed. RT-PCR was performed using the following primers: sense, 5'-CACT ACCGAGGAGCCCAGGATACAG-3'; antisense, 5'-TGCGAAAAAGTCGTAGTTCACC TGG-3'. The PCR product size was $976 \mathrm{bp}$. The products were ligated to pMD19-T simple, and the recombinant vector was sequenced.

\section{Deletion of the DNA fragment between two LoxP sites using Cre recombinase}

Cre recombinase can be used to delete the DNA fragment between two LoxP sites. First, $1 \mu \mathrm{L}$ Cre plasmid, $96 \mu \mathrm{L}$ high glucose DMEM, and $3 \mu \mathrm{L}$ liposomes were combined in a 1.5-mL Eppendorf tube, and the mixture was incubated at room temperature for $30 \mathrm{~min}$. The culture medium was removed, and the wells were washed with PBS twice, and $200 \mu \mathrm{L}$ high glucose medium was added into each well. Then, $100 \mu \mathrm{L}$ of the mixture was added into each well for $6 \mathrm{~h}$ of culture, and stably transfected cells were obtained by adding $10 \mathrm{ng} / \mathrm{mL}$ puromycin after $48 \mathrm{~h}$ of culture. The cells were normally cultured after 2 days of screening. The genomic DNA was extracted, and PCR was performed using the following primers: sense, 5'-CGCTTTCTTCCCTTCCTTTCTCG-3'; antisense, 5'-CGAAATCTCGTGATGGCAGGT 
TG-3'. The size of PCR products with neomycin resistance was $1866 \mathrm{bp}$, and the size of PCR products without neomycin resistance was $244 \mathrm{bp}$.

\section{Detection of hFat-1 expression using real-time PCR}

The primers used to amplify $\beta$-actin were as follows: sense, 5'-GGACTTCGAGCAG GAGATGG-3'; antisense, 5'- AGGAAGGAGGGCTGGAAGAG -3'. The size of the PCR product was $138 \mathrm{bp}$. The hFat-1 primers were as follows: sense, 5'-GTCAAGTCCATCCGC TATCTTGT-3'; antisense, 5'-CCGAACAGCCCGAAATACTC-3'. The size of the PCR product was $92 \mathrm{bp}$.

For real-time PCR, $10 \mu \mathrm{L}$ SYBR Green, $0.8 \mu \mathrm{L}$ sense and antisense primers, $2 \mu \mathrm{L}$ template, and $6.4 \mu \mathrm{L}$ RNase-free water were combined in an EP tube, and the PCR protocol was as follows: $95^{\circ} \mathrm{C}$ for $30 \mathrm{~s}, 40$ cycles of $95^{\circ} \mathrm{C}$ for $5 \mathrm{~s}$, and $60^{\circ} \mathrm{C}$ for $31 \mathrm{~s}$. PCR was performed using a qTOWER 2.2 Realtime PCR instrument (Analytik Jena, Jena, Germany).

Normal PFFs were used as controls, and the hFat-1 transgenic PFFs that were not treated with Cre plasmid (hFat-1-neo+) and the hFat-1 transgenic PFFs that were treated with Cre plasmid (hfat-1-neo-) were used as detection groups. Total RNA concentration was detected before reverse transcription was carried out, and equal quantities of RNA were added.

\section{High-performance gas chromatography analysis of fatty acids in transgenic PFFs}

The hFat-1-neo+, hFat-1-neo-, and PFF cells were cultured with $60 \mu \mathrm{M}$ arachidonic acid for $48 \mathrm{~h}$. Cells were then trypsinized for 3 min using $0.05 \%$ trypsin, and lipids were extracted as previously reported (Wu et al., 2012). Briefly, the cells were homogenized in a mixture of methanol, chloroform, and water. After $15 \mathrm{~min}$, chloroform was added and the samples were vortexed and centrifuged. The lower phase was dried under nitrogen and resuspended in boron trifluoride methanol. The samples were heated at $90^{\circ} \mathrm{C}$ for $30 \mathrm{~min}$ and extracted with $4.0 \mathrm{~mL}$ pentane and $1.5 \mathrm{~mL}$ water. The mixtures were vortexed and the upper phase was recovered. The extracts were dried, resuspended, and injected into a capillary column (SP-2380, $105 \mathrm{~m}$ x $953 \mathrm{~mm}$ ID, $0.20 \mu \mathrm{m}$ film thickness; Sigma). Gas chromatography was performed on an Agilent Technologies; 7890A GC System Components were identified by comparing the retention times with those of authentic standards (Sigma).

\section{Statistical analysis}

The Student $t$-test was used to determine the significance between two groups. Oneway analysis of variance with the Tukey post-hoc test was used to determine the significance between more than two groups. Statistical significance and the $\mathrm{P}$ value are indicated on the figures where appropriate. $\mathrm{P}$ values of $<0.05$ were considered to be statistically significant.

\section{RESULTS}

\section{Constuction and identification of pC-hFat-1-PGK-neo ${ }^{\mathrm{r}}$}

To identify pCAGDNA3-CAGG, eight colonies were selected, plasmids extracted from colonies No. 2 and 7 were digested with $P a c I$ and $X h o I$. Bands at 1.8 and $4.3 \mathrm{~kb}$ were obtained (Figure 1A), which suggested the enzyme sites PacI, FseI, SfiI were inserted into 
the pCAGDNA3 plasmid. The fragments LoxP-PGK-neor-BGHpA-LoxP was amplified and inserted into the pCAGDNA3-CAGG bones, the ligated product, named pC-PGK-neo ${ }^{\mathrm{r}}$-pA plasmid, was transfected and cloned, after digested with BstBI and DraIII, lanes at 1681 and 5139 bp were shown, and with HindIII and NheI, products at 1664 and 5156 bp were

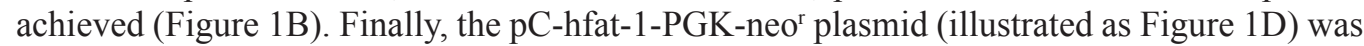
constructed followed with the inserted humanized hFat-1 sequence into pC-PGK-neo ${ }^{\mathrm{r}}$-pA, and identified with endonucleasese. The products were $8037 \mathrm{bp}$ with NheI digested, 1209 and 6828 bp with BamHI digested and 1418 and 6619 bp with XbaI (Figure 1C).
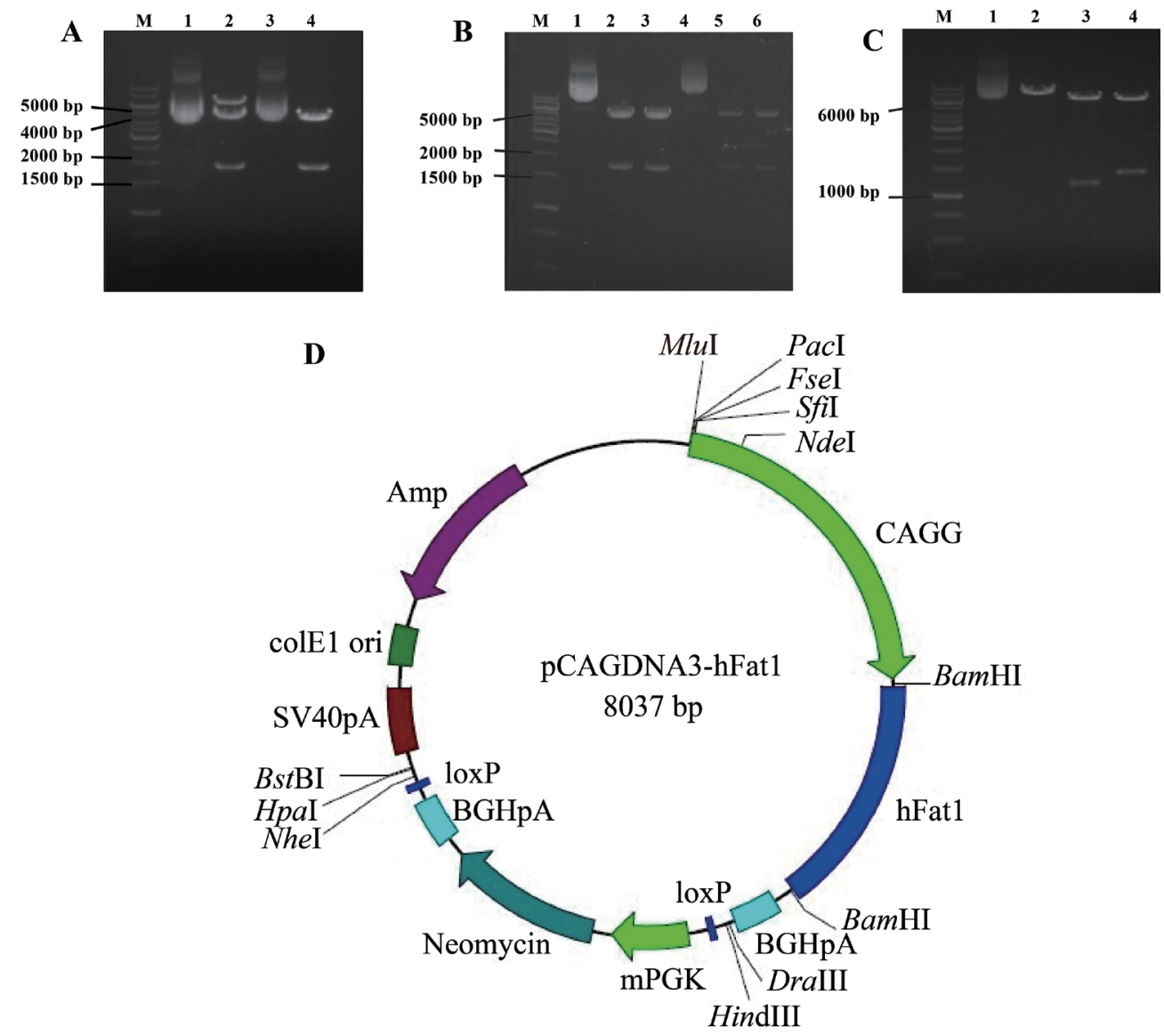

Figure 1. Construction of hFat-1 expression vector. A. Restriction endonuclease digestion of pCAGDNA3-CAGG, lane M: 1-kb DNA marker; lane 1: plasmid 2; lane 2: restriction endonuclease digestion of plasmid 2 with PacI and XhoI; lane 3: plasmid 7; lane 4: restriction endonucleases digestion of plasmid 7 with PacI and XhoI. B. Restriction endonuclease digestion of pC-PGK-neor-pA, lane $M$ : 1-kb DNA marker; lane 1: plasmid 1; lane 2: restriction endonuclease digestion of plasmid 1 with Bst $\mathrm{BI}$ and DraIII; lane 3: Restriction endonuclease digestion of plasmid 1 with HindIII and NheI; lane 4: Plasmid 2; lane 5: restriction endonuclease digestion of plasmid 2 with Bst BI and DraIII; lane 6: restriction endonuclease digestion of plasmid 2 with HindIII and NheI. C. Restriction endonuclease digestion of $\mathrm{pC}-\mathrm{PGK}-\mathrm{neo}^{\mathrm{r}}$-hfat-1, lane $M$ : 1-kb DNA marker; lane 1: plasmid 2; lane 2: restriction endonucleases digestion of plasmid 2 with NheI; lane 3: restriction endonuclease digestion of plasmid 2 with BamHI; lane 4: restriction endonuclease digestion of plasmid 2 with $\mathrm{XbaI}$. D. Map of the hfat-1 expression vector. 


\section{Identification of transgenic PFFs at DNA and RNA levels}

Three transgenic PFFs were obtained after transfection and G418 selection, and cell lines No. 2 and 3 were used for further analyses. Genomic DNA of hFat-1 transgenic PFFs was extracted, and PCR was performed to generate the hFat 1 gene. Two bands at 976 and 1349 bp were obtained (Figure 2A and B), total RNA was also extracted, and reverse transcription PCR was performed.

\section{Deletion of a PGK-neo ${ }^{\mathrm{r}}$ fragment between two LoxP sites using Cre recombinase}

Stably transfected cells were screened using $10 \mathrm{ng} / \mathrm{mL}$ puromycin following transfection with the Cre plasmid. The genomic DNA of hFat-1 cells was extracted before and after Cre plasmid transfection, and the gene was identified using PCR. A band at 1866 bp was obtained in cells that were not treated with Cre plasmid (Figure 2C), and a band at 244 bp was obtained in cells that were treated with Cre plasmid (Figure 2D), which indicated that a 1.622 kb PGK-neor fragment had been deleted by Cre recombinase.

\section{RT-PCR analysis of hFat1 expression}

Because the Fat1 gene does not exist in the pig genome, the expression of hFat-1 cannot be detected in normal PFFs. Therefore, RT-PCR data suggested that hFat-1 expression in the cells that were not treated with Cre plasmid was significantly higher than in those treated with Cre plasmid (Figure 2E).
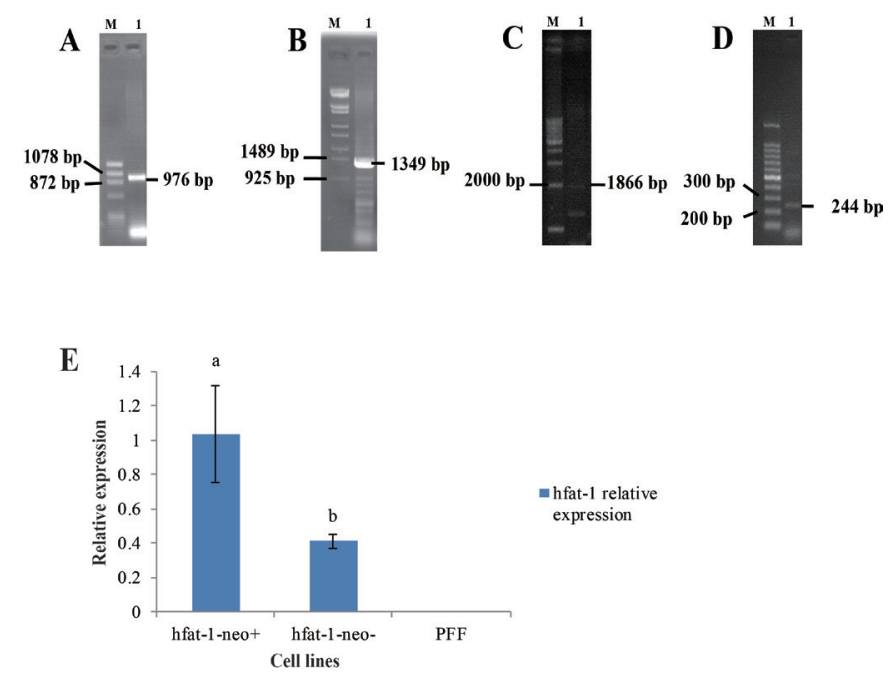

Figure 2. Identification of hFat-1 and hfat-1-neo+ porcine fetal fibroblasts and comparison of hFat-1 expression in hfat-1-neo+ and hfat-1-neo- cells. A. Identification of the hfat-1 gene in transfected porcine fetal fibroblasts by PCR, lane M: DNA marker Фx174-HaeIII; lane 1: transgenic porcine fetal fibroblasts. B. Identification of the hfat-1 gene in transfected porcine fetal fibroblast by PCR, lane M: DNA marker $\lambda \mathrm{DNA} /$ EcoT14; lane 1 : transgenic porcine fetal fibroblasts. C. Identification of hfat-1-neo+ cells by PCR, lane M: 1-kb marker; lane 1: identification of hfat-1-neo+ cells by PCR. D. Identification of hfat-1-neo- cells by PCR, lane M: 100-bp marker; lane 1: identification of the hfat1-neo- cells by PCR. E. Expression of hFat-1, as determined by real-time PCR in transgenic porcine fetal fibroblasts. 


\section{High performance gas chromatography analysis}

Two transgenic PFF cell lines were used for high performance gas chromatography analysis, and the results are shown in Tables 1 and 2 and Figure 3. Eicosapentaenoic acid (EPA) was detected in wild-type and hFat-1 transgenic cells, but was not detected in No. 2 Cre-recombinase-treated cells. The docosapentaenoic acid and DHA levels in Cre plasmidtreated hFat-1 cells were significantly higher than those in wild-type cells. The octadecadienoic acid level increased and arachidonic acid level significantly decreased in the transgenic cells (Figure 3A and B). Compared with wild-type cells, the $n-6 / n-3$ ratio significantly decreased in the transgenic cells, especially in the Cre plasmid-untreated hFat- 1 cells, suggesting that $\mathrm{hFat}-$ 1 is normally expressed in these two types of transgenic cells, and that PGK-neo ${ }^{\mathrm{r}}$ deletion can effectively elevate target gene expression (Figure $3 \mathrm{C}$ and D).

Table 1. Fatty acid content of porcine hFat-1 transgenic fibroblasts cell line No. 2.

\begin{tabular}{|c|c|c|c|c|c|c|}
\hline \multirow{2}{*}{$\frac{\text { Type of fatty acid }}{\text { ALA }(18: 3 n-3, \%)}$} & \multicolumn{2}{|c|}{ Wild type PFF } & \multicolumn{2}{|c|}{ hFat-1 transgenic PFF } & \multicolumn{2}{|c|}{ Cre recombinase treated hFat- 1 transgenic PFF } \\
\hline & - & - & - & - & - & - \\
\hline $\operatorname{EPA}(20: 5 n-3, \%)$ & 0.134 & 0.137 & 0.741 & 0.675 & - & - \\
\hline DPA $(22: 5 n-3, \%)$ & 3.015 & 2.960 & 5.031 & 4.699 & 3.364 & 2.607 \\
\hline DHA $(22: 6 n-3, \%)$ & 1.433 & 1.387 & 2.031 & 1.871 & 1.730 & 1.488 \\
\hline LA $(18: 2 n-6, \%)$ & 1.299 & 1.346 & 1.241 & 1.187 & 1.273 & 1.347 \\
\hline $\mathrm{AA}(20: 4 n-6, \%)$ & 18.823 & 20.205 & 15.962 & 16.496 & 12.545 & 9.296 \\
\hline Total n-3 (\%) & 4.582 & 4.492 & 7.803 & 7.245 & 5.094 & 4.095 \\
\hline Total n-6 (\%) & 20.122 & 21.551 & 17.209 & 17.683 & 13.818 & 10.643 \\
\hline$n-6 / n-3$ ratio & 4.39 & 4.80 & 2.21 & 2.44 & 2.71 & 2.60 \\
\hline
\end{tabular}

Table 2. Fatty acid content of porcine hFat-1 transgenic fibroblasts cell line No. 3 .

\begin{tabular}{lccccccc}
\hline Type of fatty acid & \multicolumn{2}{c}{ Wild type PFF } & \multicolumn{2}{c}{ hFat-1 transgenic PFF } & \multicolumn{2}{c}{ Crerecombinase treated hFat-1 transgenic PFF } \\
\hline ALA (18:3 n-3,\%) & - & - & 0.029 & 0.029 & 0.031 & - & - \\
EPA (20:5 n-3,\%) & 0.134 & 0.137 & 0.691 & 0.647 & 0.648 & 0.382 & 0.364 \\
DPA (22:5 n-3,\%) & 3.015 & 2.960 & 4.557 & 4.519 & 4.509 & 3.681 & 3.372 \\
DHA (22:6 n-3,\%) & 1.433 & 1.387 & 2.537 & 2.579 & 2.454 & 2.500 & 1.498 \\
LA (18:2 n-6,\%) & 1.299 & 1.346 & 0.895 & 0.966 & 1.011 & 1.058 & 1.057 \\
AA (20:4 n-6,\%) & 18.823 & 20.205 & 10.618 & 10.152 & 10.579 & 13.620 & 13.784 \\
Total n-3 (\%) & 4.582 & 4.492 & 7.814 & 7.774 & 7.642 & 14.678 & 5.234 \\
Total n-6 (\%) & 20.122 & 21.551 & 11.513 & 11.118 & 12.111 & 2.24 & 14.841 \\
n-6/n-3 ratio & 4.39 & 4.80 & 1.47 & 1.43 & 1.52 & & 2.84 \\
Mean value & & 4.60 & & & & &
\end{tabular}
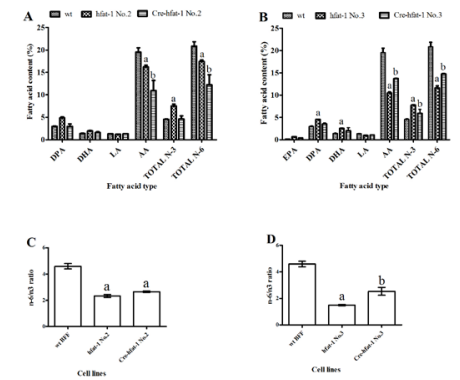

Figure 3. Fatty acid content of the hFat-1 transgenic fibroblast cell lines No. 2 and No. 3. A. B. Content of the different fatty acids in wild-type fibroblasts and the cell lines No. 2 and No. 3 before and after Cre recombinase treatment; C. D. Total n-6/n-3 fatty acid ratios of the wild type porcine fibroblasts and transgenic fibrolast cell lines No. 2 and No. 3, which are not treated and treated with Cre recombinase. 


\section{DISCUSSION}

A previous study demonstrated that the PGK promoter has dual-directional activities (Johnson and Friedmann, 1990). These authors showed that IGF-1 receptor expression decreased following the insertion of neomycin into the IGF-1R gene; hence, the postnatal proliferation of somatic cells was influenced. The abnormal RNA splicing was observed when the neomycin resistance gene was inserted. Expression of the IGF-1 receptor and the growth of heterozygote and homozygote cells decreased following neomycin insertion (Holzenberger et al., 2000). Fiering et al. (1995) performed site-directed deletion of the 5' end of DNase hypersensitive sites 2 ( $5^{\prime}$ HS2) in the $\beta$-globin locus regulation region. $5^{\prime}$ HS2 was found to be important in the regulation of $\beta$-globin expression. PGK-neo ${ }^{r}$ dramatically influenced $\beta$-globin expression. However, deletion of PGK-neo ${ }^{r}$ could recover normal $\beta$-globin expression; its transcription was affected by the presence of the resistance gene. Seidl et al. (1999) sequentially arranged $\mathrm{C} \mu, \mathrm{C} \delta, \mathrm{C} \gamma 3, \mathrm{C} \gamma 1, \mathrm{C} \gamma 2 \mathrm{~b}, \mathrm{C} \gamma 2 \mathrm{a}, \mathrm{C} \varepsilon$, and $\mathrm{C} \alpha$ from the $5^{\prime}$ to the $3^{\prime}$ end of the constant region of the Ig heavy chain . $C \delta$ expression was found to be related to class-switch recombination (CSR), and germ-line transcription (GT) occurred in the constant region of the heavy chain. In cultured B cells, insertion of PGK-neor into two sites downstream of C $\alpha$ inhibited the CSR and GT of the $120-\mathrm{kb}$ gene upstream of CH. PGK-neo insertion in the $\mathrm{I} \gamma 2 \mathrm{~b}$ locus might inhibit the CSR and GT of $\mathrm{C} \gamma 3$ upstream genes; however, this insertion did not have any obvious influence on downstream $\mathrm{C} \gamma 2 \mathrm{a}$ and $\mathrm{C} \varepsilon$ genes. Additionally, the CSR of $\mathrm{C} \gamma 3, \mathrm{C} \gamma 2 \mathrm{~b}$, and $\mathrm{C} \gamma 2 \mathrm{a}$ was inhibited by replacing the exons of $\mathrm{C} \varepsilon$ with PGK-neo ${ }^{\mathrm{r}}$ in the reverse direction. Hug et al. (1996) demonstrated that deletion of PGK-neo ${ }^{\mathrm{r}}$ was important for the phenotypic analysis of the locus control region. In subsequent research, PGK-neo ${ }^{r}$ was inserted into the intragenic HS3, and $\beta$-globin expression decreased. Pham et al. (1996) designed directional mutagenesis in two gene clusters, the telomerase $\mathrm{B}$ gene cluster and the $\beta$-like globin gene cluster. The insertion of PGK-neo ${ }^{\mathrm{r}}$ into telomerase B genes significantly decreased the expression of multiple genes, even one that was $100 \mathrm{~kb}$ away from the point of mutation. PGK-neo ${ }^{\mathrm{r}}$ abolished the expression of genes downstream of $\beta$-globin following insertion into its LCR. Target mutation of cathepsin $\mathrm{G}$ ( $3^{\prime}$ end) had a small effect on expression of the upstream telomerase gene. The mechanism of this long-distance effect is still not clear; however, PGK-neor gene expression occurs through regulation of the PGK-neo ${ }^{r}$ insertion region. These findings suggested that the PGK-neo ${ }^{\mathrm{r}}$ gene can interact with LCR, and that it can disturb the interaction between a gene locus and long-distance regulation of certain gene regions. In the present study, we generated a vector to induce exogenous expressionh of Fat-1, which was followed by the PGK-neo ${ }^{r}$ expression cassette, in PFFs. hFat-1 expressing cell lines were treated with a Cre-recombinasecontaining plasmid to remove the PGK-neo ${ }^{\mathrm{r}}$ gene. According to the results of RNA and fatty acid content analyses, the PGK-neo ${ }^{\mathrm{r}}$ gene was found to affect exogenous gene expression in a cis manner, which has been demonstrated in other studies. Deletion of the PGK-neo ${ }^{\mathrm{r}}$ expression cassette resulted in decreased expression of hFat-1 transcript and a decrease in the n- $6 / \mathrm{n}-3$ ratio; this suggests that this cassette might enhance the expression of adjacent genes.

Mammals cannot convert n-6 PUFAs into n-3 PUFAs; n-3 PUFAs must be supplied through the diet. However, the content of n-3 PUFAs is extremely low in food. Furthermore, the imbalance of n-3/n-6 may influence the development of multiple diseases, and many studies have suggested that $n-3$ PUFAs have a positive effect on diseases affecting the heart, brain, or other regions of the body. The dietary requirement of humans cannot be met by few highn-3-containing foods. However, transgenic technologies are capable of generating animals or 
plants that contain abundant n-3 PUFAs, and these provide an additional source of these nutrients for human beings. Spychalla et al. (1997) identified the Fat-1 gene in C. elegans in 1997, which was of high interest to many researchers. Kang et al. (2004) generated a Fat-1 transgenic mouse model and found that the n-6 PUFA content decreased and that of n-3 PUFA increased, and that multiple types of n-6 PUFAs were effectively transformed. Lai et al. (2006) and Li et al. (2009) optimized the generation of six Fat-1 transgenic pigs. The n-3 PUFA content was significantly elevated, and this was combined with low levels of n-6 PUFAs, and the total ratio of n-6/n-3 PUFAs was decreased 5-fold. Ji et al. (2009) generated seven founders of a Fat-1 transgenic C57/BL6 mouse colony, and Fat-1 was specifically expressed by modulating the regulatory sequence of adipocyte protein- 2 . However, it was not possible to obtain homologous offspring from two overexpressing founder Fat-1 transgenic mice, suggesting that Fat-1 overexpression may be embryonic lethal. The n-6/n-3 fatty acid ratio in serum decreased by $23 \%$ when Fat- 1 transgenic mice were fed with forage containing high levels of n- 6 unsaturated fat, and the concentration of n-3 EPA increased by $61 \%$. Moreover, the DHA content in white adipose tissue increased by $19 \%$ (Ji et al., 2009). Wu et al. (2012) generated an mFat-1 transgenic calf, and found that the n-3 PUFA content increased in all samples, whilst that of n-6 PUFAs simultaneously decreased. Interestingly, the ratios of n-6/n-3 PUFAs in tissues and milk significantly decreased (Wu et al., 2012). In China, as Fat-1 transgenic porcine model was generated in 2010 using somatic cell nuclear transfer. The sFat-1 gene was transfected into PFFs using liposomes, and the recombinant embryos were generated using somatic cell nuclear transfer. Overall, 1889 recombinant embryos were transplanted into 10 female recipients with spontaneous estrus, and 13 of 21 piglets were found to express the sFat1 gene (Pan et al., 2010). Guo et al. (2011) investigated gene expression inFat-1 transgenic and wild-type calf using cDNA chips and found that several genes were overexpressed or inhibited in Fat-1 transgenic calf, suggesting that these genes were associated with lipid metabolism, immune inflammation, neural development, and fertility. In the present study, we introduced a humanized Fat-1 gene into PFFs and obtained several viable cell lines. In these cell lines, hFat-1was expressed at a high level, and the n-6/n-3 ratio was decreased compared with that in wild-type fibroblasts. These data are consistent with previously reported findings. However, considering the safety of transgenic animals when used for food and breeding, we inserted two LoxP sites beside the PGK-neo ${ }^{\mathrm{r}}$ cassette to remove this selection gene. Although the results showed that deletion of the PGK-neor cassette led to reduced levels of exogenous hFat- 1 transcript, and subsequently a decrease in the n-6/n-3 ratio, the Cre-recombinasetreated cell lines still exhibited significantly increased $\mathrm{n}-3$ content, and might therefore provide safer material for use in transgenic breeding.

\section{Conflicts of interest}

The authors declare no conflict of interest.

\section{ACKNOWLEDGMENTS}

Research supported by the National Transgenic Project of China (\#2013ZX08010-001 and \#2014ZX08010-001), the National Natural Sciences Foundation of China (\#30871408, \#31371487 and \#81471001), the National "863" Project of China (\#2009AA10Z111), and the 
Program of Higher-level Talents of Inner Mongolia University (Xueling Li, \#30101-125129). The authors gratefully acknowledge the generous support of the Collaborative Innovation Center for Cardiovascular Disease Translational Medicine of Jiangsu Province.

\section{REFERENCES}

Branda CS and Dymecki SM (2004). Talking about a revolution: The impact of site-specific recombinases on genetic analyses in mice. Dev. Cell 6: 7-28.

Costa M, Dottori M, Ng E, Hawes SM, et al. (2005). The hESC line Envy expresses high levels of GFP in all differentiated progeny. Nat. Methods 2: 259-260.

Davis RP, Costa M, Grandela C, Holland AM, et al. (2008). A protocol for removal of antibiotic resistance cassettes from human embryonic stem cells genetically modified by homologous recombination or transgenesis. Nat. Protoc. 3: $1550-1558$

Fiering S, Epner E, Robinson K, Zhuang Y, et al. (1995). Targeted deletion of 5'HS2 of the murine beta-globin LCR reveals that it is not essential for proper regulation of the beta-globin locus. Genes Dev. 9: 2203-2213.

Guo T, Liu XF, Ding XB, Yang FF, et al. (2011). Fat-1 transgenic cattle as a model to study the function of $\omega-3$ fatty acids. Lipids Health Dis. 10: 244.

Holzenberger M, Leneuve P, Hamard G, Ducos B, et al. (2000). A targeted partial invalidation of the insulin-like growth factor I receptor gene in mice causes a postnatal growth deficit. Endocrinology 141: 2557-2566.

Hug BA, Wesselschmidt RL, Fiering S, Bender MA, et al. (1996). Analysis of mice containing a targeted deletion of betaglobin locus control region 5' hypersensitive site 3. Mol. Cell Biol. 16: 2906-2912.

Ji S, Hardy RW and Wood PA (2009). Transgenic expression of n-3 fatty acid desaturase (fat-1) in C57/BL6 mice: Effects on glucose homeostasis and body weight. J. Cell Biochem. 107: 809-817.

Johnson P and Friedmann T (1990). Limited bidirectional activity of two housekeeping gene promoters: human HPRT and PGK. Gene 88: 207-213.

Kang JX, Wang J, Wu L and Kang ZB (2004). Transgenic mice: fat-1 mice convert n-6 to n-3 fatty acids. Nature 427: 504.

Lai L, Kang JX, Li R, Wang JD, et al. (2006). Generation of cloned transgenic pigs rich in omega-3 fatty acids. Nat. Biotechnol. 24: 435-436.

Li R, Murphy CN, Spate L, Wax D, et al. (2009). Production of piglets after cryopreservation of embryos using a centrifugation-based method for delipation without micromanipulation. Biol. Reprod. 80: 563-571.

Pan D, Zhang L, Zhou Y, Chong F, et al. (2010). Efficient production of omega-3 fatty acid desaturase (sFat-1)-transgenic pigs by somatic cell nuclear transfer. Sci. China Life Sci. 53: 517-523.

Pham CT, MacIvor DM, Hug BA, Heusel JW, et al. (1996). Long-range disruption of gene expression by a selectable marker cassette. Proc. Natl. Acad. Sci. U. S. A. 93: 13090-13095.

Scacheri PC, Crabtree JS, Novotny EA, Garrett-Beal L, et al. (2001). Bidirectional transcriptional activity of PGKneomycin and unexpected embryonic lethality in heterozygote chimeric knockout mice. Genesis 30: 259-263.

Schnutgen F, Stewart AF, von Melchner H and Anastassiadis K (2006). Engineering embryonic stem cells with recombinase systems. Methods Enzymol. 420: 100-136.

Seidl KJ, Manis JP, Bottaro A, Zhang J, et al. (1999). Position-dependent inhibition of class-switch recombination by PGK-neor cassettes inserted into the immunoglobulin heavy chain constant region locus. Proc. Natl. Acad. Sci. U. S. A. 96: 3000-3005.

Spychalla JP, Kinney AJ and Browse J (1997). Identification of an animal omega-3 fatty acid desaturase by heterologous expression in Arabidopsis. Proc. Natl. Acad. Sci. U. S. A. 94: 1142-1147.

Wu X, Ouyang H, Duan B, Pang D, et al. (2012). Production of cloned transgenic cow expressing omega-3 fatty acids. Transgenic. Res. 21: 537-543. 